\title{
Summary Talk: Particle Physics Phenomenology
}

\author{
Erasmo Ferreira \\ Instituto de Fisica, U.F.R.J., \\ Rio de Janeiro, RJ, 21945-970, Brasil \\ E-mail: erasmo@if.ufrj.br
}

Received on 25 February, 2001

\begin{abstract}
We summarize the contributed papers and the talks in the plenary and parallel sessions in this XXI Brazilian National Meeting on Particles and Fields that are classified as of phenomenology kind. We try to put the activities here developed in the perspective of the existing community of phenomenologists in Brazil 2000.
\end{abstract}

We recall that the phenomenology of the interaction of elementary particles was probably our main area of reasearch in the time when physics research in Brazil began, in the 1940, 1950 decades. It is interesting to remark that at those times there was fair amount of experimental activity in particle physics in Brazil (cosmic rays, nuclear emulsions), and there was also the remarkable work of Cesar Lattes in Bristol and in the Chicago accelerator. Since then, for some decades, there was less experimental particle physics than there should be, and correspondingly theoretical phenomenology developed slowly in the country. It was not at all easy to work in phenomenology in a place like Brazil before the internet came to solve the information/communication problem. Experimental data and theoretical ideas could usually take several months to reach physicists here.

Some decades ago the elementary particles were the mesons, nucleons, eletrons, photons ..., and the study of their interactions were the concern of elementary particle physics. QED succeeded beautifully, and the structure of the weak interactions was understood, while the strong interactions remained for several decades as an unsolved problem, in spite of the brilliant description of the hadron spectroscopy through $\mathrm{SU}(3)$.

Then the energies went up, and the investigations of the eletroweak properties of heavy quarks and leptons and of intermediate vector bosons took the center of the stage and became the definition of elementary particle physics research, the standard model being investigated thoroughly. The Higgs boson, its only undiscovered building block, seems to be around the corner, according to experiments described in this meeting. Brazilian physicists have been working actively in both experimental and theoretical aspects of these investigations, as exhibited in the present meeting.

The strong interactions, with the difficulties caused by the confinement and the non-perturbative aspects of QCD, poses big problems in calculations of hadron production and hadronic interactions. Methods and concepts must be developed to face these questions. The fundamental properties of QCD (vacuum structure, mechanisms of confinement, behaviour of matter at high densities and high temperatures) are still mysterious. The specificity of the problems creates the area called hadron physics (the study of hadronic structures and interactions).

The biggest electron machine LEP is now closed, its place being taken over by LHC, that together with Fermilab Tevatron and RHIC at BNL will form a group of hadronic machines where the studies of the fundamental interactions and the search for fundamental constituents will be made. Besides them, there will be the $\mathrm{e}^{+} \mathrm{e}^{-}$colliders dedicated mainly to b-physics.

It is remarkable that Hadron Physics is well organized in Brazil, with a strong and active community that holds regular meetings of both national and international scope. Only a small part of this community participate regularly in ENFPC. With the changes in the frontiers of particle physics research described above, it is important that more communication and more merging develops between the areas.

The amount of particle physics research presented in the hadronic meetings could enrich substantially the programs of ENFPC, and effort must be made in this direction.

On the other hand, the interface between particle and nuclear physics, in large part provided by hadronic physics, must be incentivated in Brazil. Modern Nuclear Physics must be QCD based, which requires rather profound changes in the research that is traditionally made in our laboratories in Brazil. The interface between particle and nuclear physics, provided by 
hadronic physics must be developed to impulsionate these changes.

\section{The Plenary Sessions}

PL1 - Maria Beatriz Gay Ducati, UFRGS, Brazil : High Density QCD.

PL2 - Jonathan L. Rosner, University of Chicago, USA : CP Violation: Past, Present and Future.

The Parallel Sessions

PA1 - Gastão Krein, IFT/Unesp, Brazil : The Gerasimov-Drell-Hearn Sum Rule and the Spin Structure of the Proton.

PA2 - Marcelo M. Guzzo, UNICAMP, Brazil : New Paradigms in Neutrino Physics.

I find that the organizing committee was wise and lucky in choosing these topics for the plenary and parallel sessions in phenomenology.

M.B. Gay Ducati described the work of hers and her students on the difficult dynamics of QCD in the confinement regime. Including unitarity and screening corrections in the evolution equations, they obtain new equations, which are then solved with approximations, to obtain observable quantities of the proton structure. The screening corrections will be particularly important in the analysis of the heavy ion and hadronic collisions that will be observed in future accelerators.

J.L. Rosner presented a beautiful and competent review of the studies of $\mathrm{CP}$ violation that date since the first discovery of neutral $\mathrm{K}^{0}$ mesons decaying in wrong $\mathrm{CP}$ states. The violation of $\mathrm{CP}$ symmetry, which is related to the behaviour of systems under time reversal, is now under intense scrutiny by experimentalists and theorists, who try to understand all aspects of the systems and dynamics involved. The studies will clarify the parameters of the standard model and the dynamics of the electroweak interaction. The $\mathrm{B}$ mesons produced in Fermilab and in the b factories in Cornell, SLAC and KEK and the complex experiments to study $\mathrm{K}$ decays in CERN and Fermilab are giving extensive and accurate information for this fundamental area of particle physics.

In spite of the frightening title (how many people knew about the GDH sum rule, with all its simplicity and exactness?) the talk by Gastão Krein was pedagogically very clear and stimulating. Interesting to hear how the low energy photon experiments can test the proton structure, complementing the studies with deep inelastic scattering made in the high energy machines. It was nice to hear about the Ragusa polarizabilities (Silvestre Ragusa, our colleague from São Carlos), which were introduced many years ago and are a useful tool in these studies.

Neutrino physics has been a very hot subject in the recent years, with several problems and intriguing questions, and a dramatic effect in our knowledge of the fundamental particles, due to the discovery of the non-zero neutrino masses and the neutrino oscillations. The discoveries are important also for cosmology as for the study of the physics processes inside the sun. The number and nature (may some of them be of Majorana kind?) of the neutrinos, and their mass spectrum will be investigated for several years to come. The talk by Guzzo was a very elucidating and updated review of the situation.

\section{Oral Presentations in Phenomenology}

ORAL01 : SUSY: radiative effects on squark production.

ORAL02 : SUSY: signatures for R-parity violation.

ORAL03 : Beyond standard model: signatures for extra large dimensions.

ORAL04 : Solar neutrino problem solution with spinflavor precession.

ORAL05 : Solar neutrino problem solution with longwavelength neutrino oscillations.

ORAL06 : Neutrino oscillations due to quantum decoherence.

ORAL07 : Beyond the standard model: exotic bosons of charge 2 decaying into 2 leptons in 3-3-1 model.

ORAL08 : Neutrino mass oscillations formulae more carefully evaluated with wavepackets formed in the creation process.

ORAL 09 : Nambu-Jona-Lasinio model in a renormalizable version to evaluate mesonic observables.

ORAL10 : SUSY : leptoquarks effects in Drell-Yan processes.

ORAL11 : Beyond the standard model: CP violation described by new operators.

ORAL12 : Beyond the standard model: production of charged heavy leptons.

\section{Posters in Phenomenology}

P066 : breaking of chiral symmetry due to infrared divergence in the gluon propagator.

P067 : possibility of $\tau$ neutrino detection in the showers to be observed in Auger experiment.

P068 : spacial gluon distribution inside the proton and its influence in the observed gluon structure function.

P069 : charmonium production as a mixture of the perturbative and the (smaller) nonperturbative contributions.

P070 : soliton (skyrmion) configurations studied with three possible forms of stabilizing terms added in the chiral nonlinear sigma model.

P071 : Nambu-Jona Lasinio model to study spin 1 (vector and axial) mesons, their masses, decay constants and symmetry breaking parameters. 
P072 : complex (real plus quaternionic) potentials in nonrelativistic quantum mechanics describe decay processes, with time reversal non-invariance and thus possible connection to $\mathrm{CP}$ violation.

P073 : potential models to describe heavy quarkonia bound states, with evaluation of mass spectrum and transition rates.

P074 : analysis of pp cross-sections at high (cosmic ray) energies $(\sqrt{s} i 10 \mathrm{GeV})$ from different predictions and evaluation of the real part $\rho$ parameter using dispersion relations.

P075 : study of the representation of elastic differential cross sections by profile functions of gaussian form.

P076 : Nambu-Jona-Lasinio model with parametrized constituent masses to study mesonic observables.

P077 : Flux of nuclei in primary cosmic rays as origin of observed hadronic and electromagnetic intensities.

P078 : Beyond the standard model: form factors for couplings of 3 neutral bosons.

P079 : Vector meson photoproduction described by superposition of hard and soft contributions.

P080 : Perturbative QCD calculation (exchange of gluon ladders) of pp and $r \bar{m} p$ p cross sections.

P081 : Analysis of solar neutrinos observed in SuperKamiokand experiment, with determination of masses and oscillation parameters.

P082 : Diffusion of hadronic fluxes from cosmic rays in the atmosphere.

P083 : Beyond the standard model : effects of a heavy exotic lepton in the $e^{+} e^{-} \rightarrow 2 Z^{0}$ process.

P084 : QCD effects of dynamical gluon mass in hadronic decays of heavy vector mesons.

P085 : QCD nuclear (deuterium) effects in the determination of parton densities.

P086 : Beyond the standard model: supergravity model leads to scalar and pseudoscalar particles coupled to photons that affect cosmological observables.

P087 : Nambu-Jona-Lasinio model with analytical extension of the 4-point function that produces quark confinement.

P088 : Quark model determination of mixing angles in mesonic nonets.

P089 : Standard model calculation of $\tau \rightarrow \pi \pi \pi \nu_{\tau}$ with effective hadronic corrections.

P090 : Spin asymmetries in the final states in polarized proton-proton collisions.

P091 : Experimental: Fluorescence detector for the Auger Observatory.

P092 : Experimental : separation between electromagnetic and muonic components in observed showers in the Auger experiment.

P093 : Experimental : Fluctuations in composition of showers in Auger experiment.

P094 : Solar neutrino problem with the spin-flavor precession solution.

P095 : Neutrino interactions with flavor changing in future neutrino factories.

P096 : Model with composite quarks.

P097 : Effective chiral lagrangian for NN interaction with mass dependence in central force component.
P098 : QCD effects in photoproduction of vector mesons.

P099 : Strangeness content in nucleon structure.

P100 : Beyond standard model : production of new neutral leptons.

P101 : Crossing symmetry violation in chiral perturbation theory.

\section{Submitted Contributions in Phenomenology}

$$
12 \text { Oral + } 36 \text { Posters }
$$

Distibution of Subjects

- Physics Outside the Standard Model

Susy Tests : 4

Other Models : 6

- Strong Interaction, Low Energies

Effective, Chiral, Skyrmions : 5

Models (NJL) : 4

- Strong Interactions, High Energies QCD (Perturbative+Nonperturbative) : 7

Quark and Potential Models : 4

Hadronic Collisions : 2

- Neutrinos

Solar $\nu$ Problem : 5

$\nu$ Oscillation Effects : 5

- CP Violation : 2

- Cosmic Rays

Models for C.R. Fluxes : 2

Experimental (Auger) : 3

- Structure of Quarks : 1

\section{What is Missing - and should be here}

- Precision Tests of the Standard Model

- Determination of CKM Matrix Elements

- Heavy Flavours - matrix elements, form factors, decay rates.

- Quark-gluon and nuclear matter.

Research is made in these areas in Brazil, as can be seen in other meetings that were held in Brazil in the year 2000: Hadron Physics, RANP, Heavy Quarks with Fixed Targets. 